\title{
Handling unfavourable polarization states in a polarization-based shearing speckle interferometer
}

\author{
Peter A A M Somers and Nandini Bhattacharya \\ Optics Research Group, Delft University of Technology, Lorentzweg 1, NL-2628 CJ Delft, \\ The Netherlands
}

E-mail: P.A.A.M.Somers@TUDelft.nl and N.Bhattacharya@TUDelft.nl

Received 15 December 2006, accepted for publication 11 April 2007

Published 22 May 2007

Online at stacks.iop.org/JOptA/9/S92

\begin{abstract}
A polarization-based shearing speckle interferometer requires the incoming scattered light to have proper polarization characteristics: the light should be equally distributed over two orthogonal polarization states in order to obtain two interfering beams with equal intensities and the highest possible modulation. Many surfaces scatter linearly polarized light randomly, providing equal intensities for the interfering beams on average. However, when a diffusely reflecting metallic surface is illuminated by a linearly polarized laser beam, the polarization direction of the illuminating beam is retained to a large extent in the scattered light. As a result light entering the interferometer will not be randomly polarized, which may lead to low modulation due to unbalanced object and reference beams. A solution that handles the problem effectively is proposed. It consists of a quarter-wave plate positioned in front of the interferometer, oriented at $45^{\circ}$. It is shown that unfavourable predominant polarization states encountered when testing unprepared metallic surfaces can be converted into favourable ones, thereby obtaining well balanced object and reference beams, irrespective of the polarization direction of the incoming light.
\end{abstract}

Keywords: speckle interferometry, shearography, polarization, surface preparation

(Some figures in this article are in colour only in the electronic version)

\section{Introduction}

Speckle interferometry is a commonly used technique for the measurement of small displacements in a wide range of applications. A special category of this technique is shearography, characterized by the fact that the two interfering wavefronts both originate from the object being tested. This set-up provides intrinsic stability against environmental disturbances since both beams travel along almost the same optical path. For this reason shearography is an obvious choice in non-destructive testing when small defect-related displacements must be measured in industrial environments. Especially for this kind of application it is highly desirable that objects can be inspected without surface preparation.

Polarization-based shearing speckle interferometers make use of a polarization-sensitive element to create two orthogonally polarized beams, one of them sheared, both originating from the object of interest. The beams interfere at a CCD sensor after passing an analyser. Possible implementations for the polarization-sensitive element are a Wollaston prism [1-3], a Savart plate [4], a birefringent wedge [5], or another beamsplitting device such as a polarizing beamsplitter cube, used with two mirrors in a Michelson configuration $[6,7]$. One of the mirrors can be tilted to produce a variable shearing angle between the two beams.

When two speckle patterns interfere, the intensity $I$ at any pixel of the camera is given by the interference equation (pixel coordinates are omitted for clarity):

$$
I=I_{\mathrm{o}}+I_{\mathrm{r}}+2 \sqrt{I_{\mathrm{o}} I_{\mathrm{r}}} \cos \varphi,
$$

where $I_{\mathrm{o}}$ and $I_{\mathrm{r}}$ are the intensities of the object and reference 
beams and $\varphi$ is the phase difference between the two interfering beams at that particular location. The modulation term

$$
I_{\mathrm{M}}=2 \sqrt{I_{\mathrm{o}} I_{\mathrm{r}}}
$$

reaches its highest value when the interfering beams have equal intensities. Modulation is zero if one or both of the interfering beams have zero intensity. High modulation is desirable for high $\mathrm{S} / \mathrm{N}$ ratios, a condition that cannot always be met in actual situations, as will be discussed in the next section.

\section{Problem description}

Since the intensity distributions of both interfering speckle patterns have their maximum at zero intensity [8], modulation is zero or low at many locations, resulting in poor signalto-noise ratios. Low modulation can also occur due to unbalanced interfering beams, caused by unfavourable polarization conditions. It is therefore desirable that the polarization state of incoming light is well defined, for instance by taking care that incoming light is linearly polarized at $45^{\circ}$ upon entry.

The interferometer acquires speckle patterns that are generated by a scattering object illuminated by a uniform beam of coherent light. The illumination is linearly polarized in general. The state of polarization of the scattered light is determined by the properties of the scattering object. Some materials are strongly depolarizing, producing randomly polarized scattered light that enters the interferometer. Although not ideal, this situation is acceptable when the average intensities of the interfering beams are the same. Metallic materials, however, show very low levels of depolarization [9]. As a result the scattered field may have an unfavourable predominant polarization state, depending on the polarization of the illuminating beam.

There are a number of measures that can be taken to solve this problem. First of all, the properties of the scattering object might be changed by covering it with a layer that produces stronger depolarization of the incoming light. This solution is often undesirable for industrial applications.

Secondly the polarization state of the illumination could be altered, for instance by rotating the polarization direction of linearly polarized illumination, or by converting the polarization state of the light to circular. However, partly reflecting or scattering surfaces may show different reflective properties for s- and p-polarized light, causing a predominant linear polarization state in the scattered field, even when both horizontal and vertical states are equally present in the illumination [10]. This effect also depends on the geometry of the illuminated object. Moreover, when using more than one illumination system, every single system should be modified.

A third approach could be based on a change of the polarization state of the scattered light, just before it enters the interferometer. A polarizer oriented at $45^{\circ}$ establishes a 1:1 relation between horizontal and vertical polarization at the expense of losses for other polarization directions, in particular for $-45^{\circ}$. In addition there are absorption losses when using a polarizer. Another solution, without substantial absorption losses, is an adjustable half-wave plate that can be given the best orientation to obtain mainly $45^{\circ}$ polarized light, when the depolarizing properties of the object are not sufficient to provide random polarization. This approach requires readjustment of the half-wave plate whenever the polarization state of the scattered light makes it necessary. Furthermore it would be desirable in this case to have available some measurement system that can determine the optimum setting during adjustments.

\section{Polarization state conversion}

We have chosen another way of conditioning the light before it enters the interferometer. Our solution comprises a quarterwave plate positioned in front of the interferometer, oriented at $45^{\circ}$. The quarter-wave plate converts linearly polarized light to an elliptical state establishing a 1:1 distribution over the horizontal and vertical polarization directions. The proposed method does not require frequent readjustments and associated measurements, thus simplifying the operational use of the interferometer. Although for this system there are also polarization states that are unfavourable after conversion, the predominant state is now a favourable one. The proposed solution provides considerable improvements in practical situations, in particular for metallic objects that do not produce a depolarized scattered field when illuminated by linearly polarized light with a possibly unfavourable orientation.

The task of a device that must reduce the dependence of the interferometer on the polarization direction of incoming light is to equally distribute the optical energy present in an unfavourable linear state over two mutually orthogonal states: the unfavourable predominant polarization state will then be converted to a favourable one. The most unfavourable polarization states are either horizontal or vertical. These states are converted to circular by the quarter-wave plate. In general the quarter-wave plate converts linearly polarized light in an arbitrary direction into elliptically polarized light. The main axis of the ellipse is oriented at $+45^{\circ}$ or $-45^{\circ}$, along the fast or slow axis of the quarter-wave plate, establishing the required equal distribution of light over the horizontal and vertical polarization states. The plate will not affect the most favourable input state for an unmodified system: the state of linearly polarized light at $+45^{\circ}$ or $-45^{\circ}$ will not change. Circular states, however, will be converted to either a vertical or a horizontal one, which implies degradation for these states. Other states, in general all elliptical states, will be less affected: since elliptically polarized light can be considered as the superposition of linearly and circularly polarized light, only the circular portion gets the unfavourable vertical or horizontal state after passing the quarter-wave plate. The linear part is converted into the ideal elliptical state, the axis of the ellipse being oriented at $+45^{\circ}$ or $-45^{\circ}$.

\section{Jones' calculation}

The conversion of polarization states can be described mathematically using Jones' calculus $[11,12]$. Using

$$
V_{\alpha}=\left[\begin{array}{l}
\cos \alpha \\
\sin \alpha
\end{array}\right]
$$

for linearly polarized light in arbitrary direction $\alpha$ and

$$
M_{\mathrm{QWP} 45}=\frac{1}{2}\left[\begin{array}{cc}
1 & -\mathrm{i} \\
-\mathrm{i} & 1
\end{array}\right]
$$




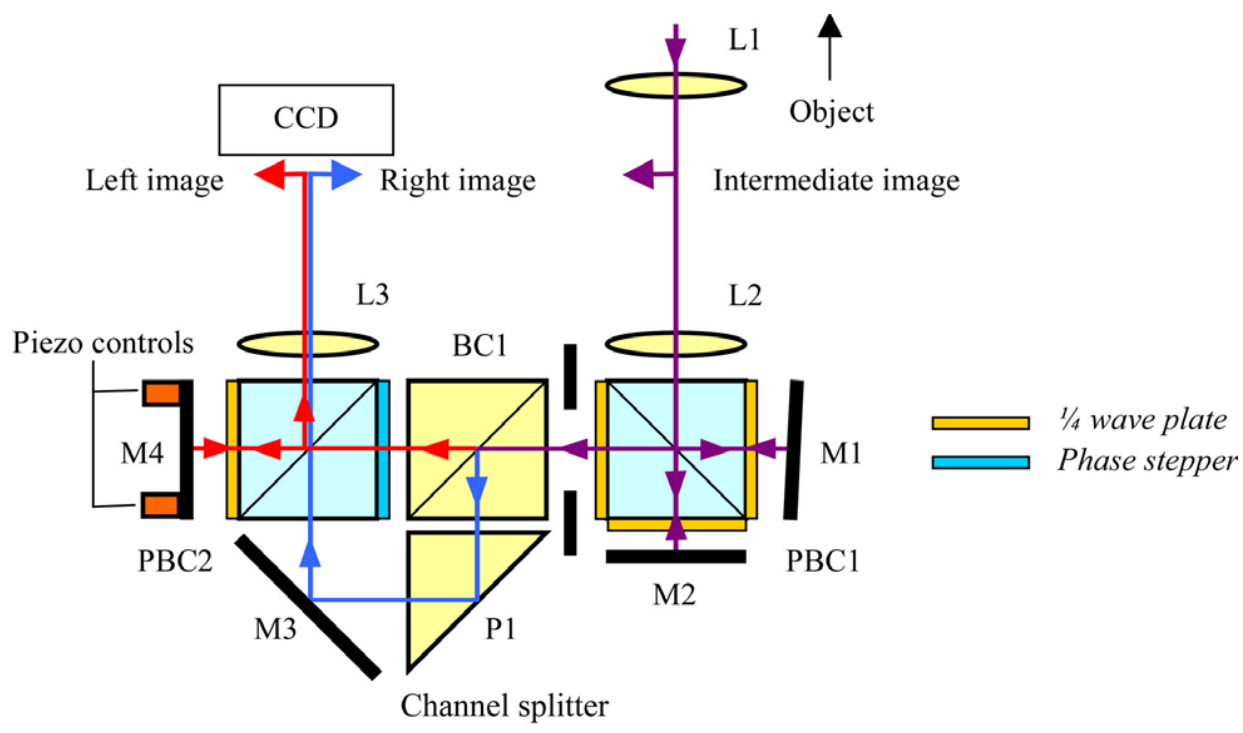

Beam combiner

Shearing unit

Figure 1. Polarization-based shearing speckle interferometer.

to represent a quarter-wave plate oriented at $45^{\circ}$, the properties of polarized light with arbitrary linear polarization, after passing a quarter-wave plate oriented at $45^{\circ}$ can be described by the Jones matrix operation:

$$
\begin{aligned}
& M_{\mathrm{QWP} 45} V_{\alpha}=\frac{1}{2}\left[\begin{array}{cc}
1 & -\mathrm{i} \\
-\mathrm{i} & 1
\end{array}\right]\left[\begin{array}{c}
\cos \alpha \\
\sin \alpha
\end{array}\right] \\
& =\frac{1}{2}\left[\begin{array}{c}
\cos \alpha-\mathrm{i} \sin \alpha \\
-\mathrm{i} \cos \alpha+\sin \alpha
\end{array}\right]=\frac{1}{2}\left[\begin{array}{c}
\mathrm{e}^{-\mathrm{i} \alpha} \\
-\mathrm{ie}^{\mathrm{i} \alpha}
\end{array}\right] .
\end{aligned}
$$

The resulting vector represents elliptically polarized light. The horizontal and vertical components have the same amplitude, so the main axis of the ellipse is oriented at $45^{\circ}$. Thus an equal distribution of incoming light is established over the two orthogonally polarized outputs, acting as object and reference beams in the interferometer. There is a phase difference between the horizontal and vertical components of the elliptically polarized light, depending on the polarization direction $\alpha$ of the incoming light. The actual phase difference at the sensor also depends on the nature of the incoming field. In our case this is a speckle field, and the phase difference between the interfering beams is random over the CCD sensor positions. However, the actual phase difference between object and reference at a particular pixel is not relevant since displacement related phase differences are the quantity of interest.

\section{Implementation}

The improvements have been implemented in an existing polarization-based shearing speckle interferometer, shown in figure 1. Light entering the interferometer is split by polarizing beamsplitter cube PBC1. Horizontally polarized light is transmitted to fixed mirror M2, and reflected back towards the beamsplitter. Passing twice a quarter-wave plate oriented at $45^{\circ}$, between mirror M2 and the beamsplitter, the horizontally polarized light is converted to vertical. The beam is then reflected by PBC1 to its exit. Similarly vertically polarized light entering the interferometer is reflected towards shearing mirror M1, reflected back and converted to the horizontal state by another quarter-wave plate. Mirror M1 is slightly tilted to obtain a sheared image. At the exit of the cube now two orthogonally polarized beams propagate into a channel splitter section that splits the beam pair into two channels to allow for a phase step in one of the channels.

The two linearly polarized beams are converted to a circularly polarized beam pair by another quarter-wave plate attached to the exit of $\mathrm{PBC} 1$, oriented at $45^{\circ}$. One beam is converted to left-circularly polarized light, the other to rightcircularly polarized light. The channel splitter consisting of a non-polarizing beamsplitter $\mathrm{BC} 1$ and a $45^{\circ}$ prism $\mathrm{P} 1$ splits the circularly polarized beam pair into two pairs. A $\pi / 2$ phase step is implemented in one of the channels by a polarization plane rotator, attached to polarizing beamsplitter $\mathrm{PBC} 2$ that is used as a beam combiner. Mirrors M3 and M4 are set to a position for which the imaging system projects two images of the object side by side onto the CCD camera. Mirror M4 can be fine-adjusted by piezo-elements for sub-pixel alignment of the two images [13]. As a result two speckle interferograms are obtained side by side on a single CCD camera, with a mutual phase step of $\pi / 2$. A more detailed description of the interferometer, including the mathematical treatment of the phase stepping method, can be found in [7, 14].

The modification of the interferometer is implemented by inserting a quarter-wave plate oriented at $45^{\circ}$ in front of beamsplitter PBC1. The quarter-wave plate converts linearly polarized light in any direction into an elliptical state as presented in section 4, equation (3), establishing an equal distribution of light over the two polarization states at the exit of PBC1. The elliptically polarized light is split by $\mathrm{PBC} 1$ into a horizontally and a vertically polarized part, $V_{\mathrm{HOR}}$ and $V_{\mathrm{VERT}}$, respectively:

$$
V_{\mathrm{HOR}}=\frac{1}{2}\left[\begin{array}{ll}
1 & 0 \\
0 & 0
\end{array}\right]\left[\begin{array}{l}
\mathrm{e}^{-\mathrm{i} \alpha} \\
-\mathrm{i} \mathrm{e}^{\mathrm{i} \alpha}
\end{array}\right]=\frac{\mathrm{e}^{-\mathrm{i} \alpha}}{2}\left[\begin{array}{l}
1 \\
0
\end{array}\right]
$$


Handling unfavourable polarization states in a polarization-based shearing speckle interferometer

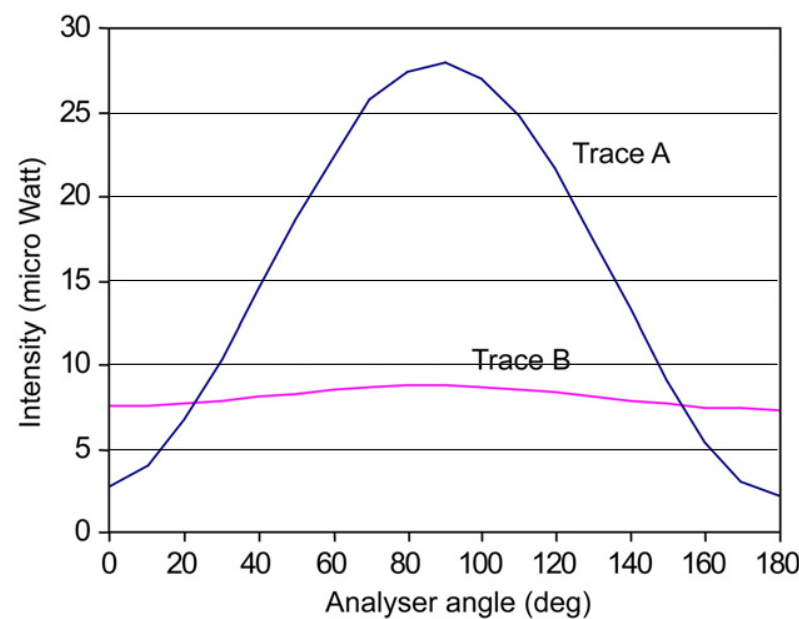

Figure 2. Intensity of light scattered by an object illuminated with vertically polarized light, as a function of analyser angle. Trace A: light scattered by a metallic surface covered with a thin primer layer. Trace B: light scattered by a surface covered with white matt paint.

$$
V_{\text {VERT }}=\frac{1}{2}\left[\begin{array}{ll}
0 & 0 \\
0 & 1
\end{array}\right]\left[\begin{array}{c}
\mathrm{e}^{-\mathrm{i} \alpha} \\
-\mathrm{ie}^{\mathrm{i} \alpha}
\end{array}\right]=\frac{-\mathrm{ie}^{\mathrm{i} \alpha}}{2}\left[\begin{array}{l}
0 \\
1
\end{array}\right] .
$$

Again, obviously, the horizontal and vertical results have the same amplitude and different phase: phase terms $\mathrm{e}^{-\mathrm{i} \alpha}$ and $-\mathrm{i}^{\mathrm{i} \alpha}$ for horizontally and vertically polarized components depend on the polarization direction $\alpha$ of incoming linearly polarized light. Similarly phase difference and amplitude are also maintained during subsequent conversion into counterrotating circularly polarized beams by the quarter-wave plate attached to the exit of PBC1.

\section{Experimental details}

Experiments have been carried out to evaluate the properties of the interferometer before and after modifying it by adding a quarter-wave plate oriented at $45^{\circ}$ in front of it. First of all a measurement was carried out on a metallic object demonstrating the problem. The object, coated by a thin primer layer, was illuminated by vertically polarized light. The polarization state of the scattered light was measured using an analyser in different angular positions. Although coated, the metallic object still scatters light with predominant vertical polarization as figure 2 clearly shows. An object that had been given the usual surface preparation, spraying it with white matt powder paint, also illuminated by vertically polarized light shows almost no dependence on analyser angle.

Another result is shown in figure 3. Here the interferometer itself is used as the measuring device. Either mirror M1 or M2 is blocked, and the average intensity of the speckle pattern on the CCD is measured as a function of the orientation of a half-wave plate, positioned in front of the interferometer. The tracks for mirror M1 and M2 presented in figure 3 show large fluctuations, due to the mainly vertical polarization of light scattered by the metallic object.

After insertion of a quarter-wave plate oriented at $45^{\circ}$, results have considerably improved, as figure 4 shows. Again the interferometer is used to measure average intensities as a function of the orientation of the half-wave plate, when either

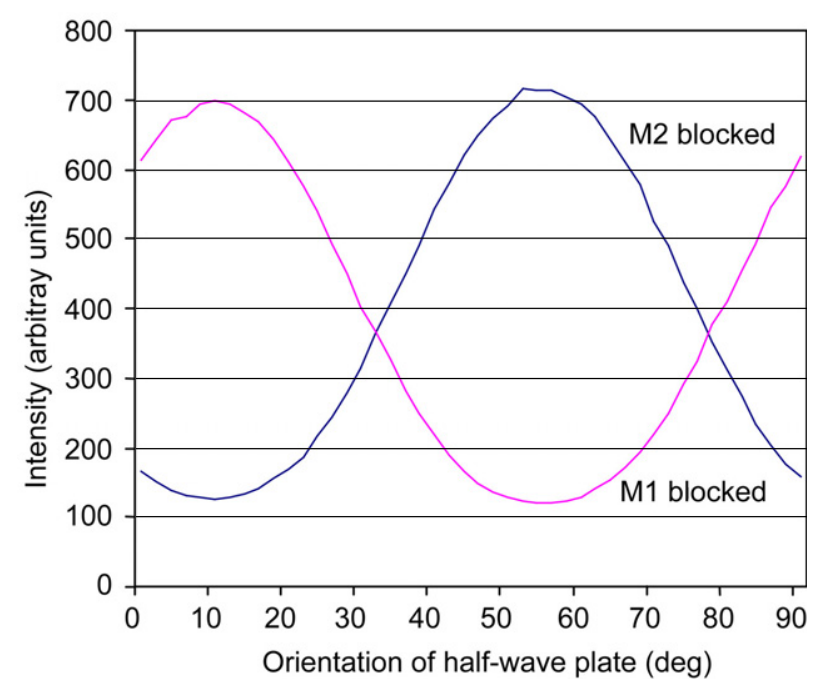

Figure 3. Intensity of light scattered by a metallic object, measured by the interferometer with either mirror M1 or M2 blocked, as a function of the polarization direction of incoming light. Polarization is changed by adjusting the orientation of a half-wave plate in front of the interferometer.

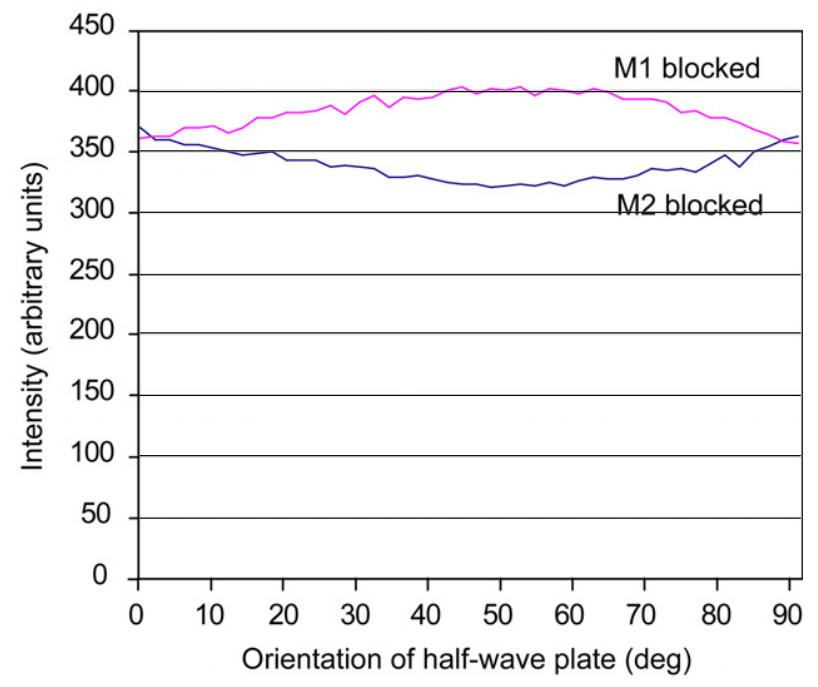

Figure 4. Intensity of light scattered by a metallic object, measured by the interferometer with either mirror M1 or M2 blocked, as a function of the polarization direction of incoming light. Polarization is changed by adjusting the orientation of a half-wave plate in front of the interferometer. The interferometer is modified by positioning a quarter-wave plate oriented at $45^{\circ}$ in front of it.

one of the mirrors M1, M2 is blocked. Some dependence remains, caused by non-ideal properties of the wave plate and its alignment.

Finally some experiments have been carried out on a component with artificial defects, before and after the modification. The measurement set-up is shown in figure 5 .

The component, a Fokker 100 speed brake containing an artificial defect with a diameter of $25 \mathrm{~mm}$, was thermally loaded to induce local deformation at the defect area. The coating of the component was removed locally in order to evaluate the performance of the interferometer on an 


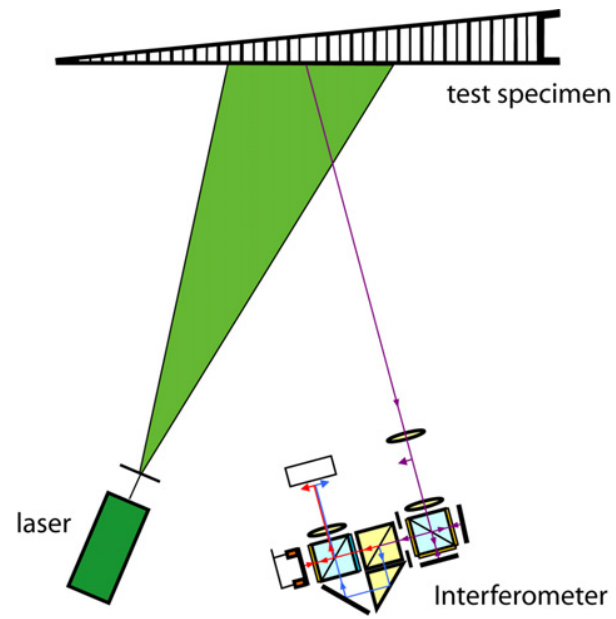

Figure 5. Measurement set-up.
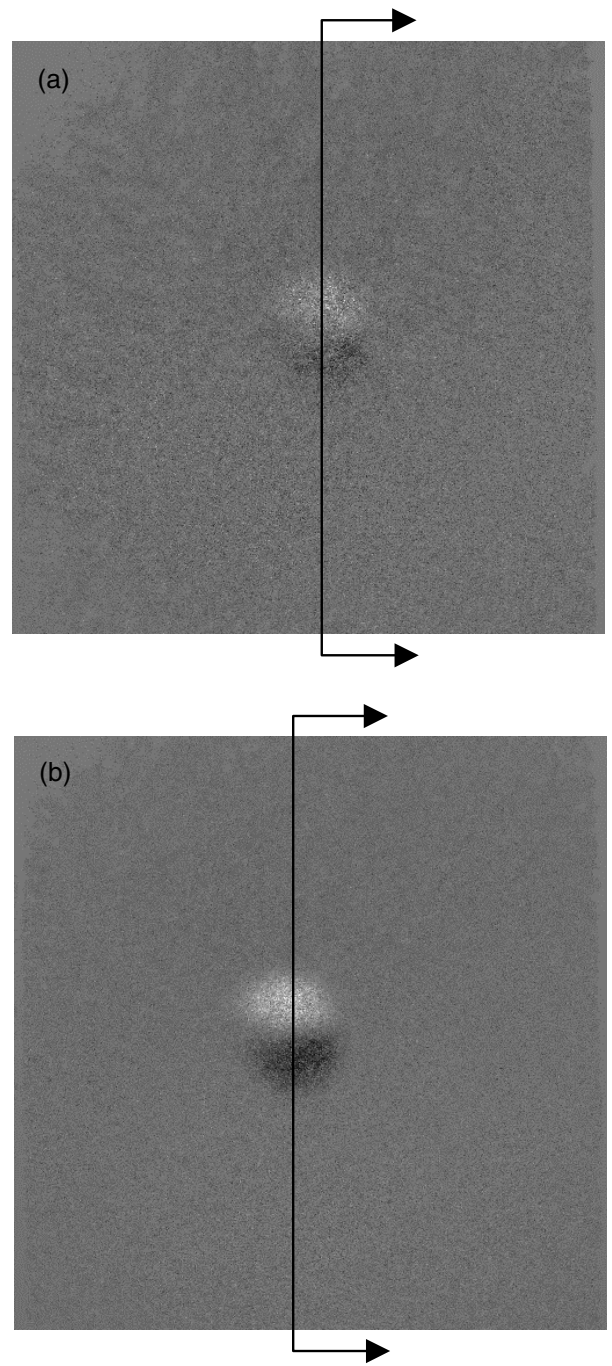

Figure 6. Indication of a defect in a Fokker 100 airbrake, after thermal loading. Results are shown (a) before and (b) after modifying the interferometer

unprepared metallic surface. The object was observed with the interferometer during cooling down, using vertical
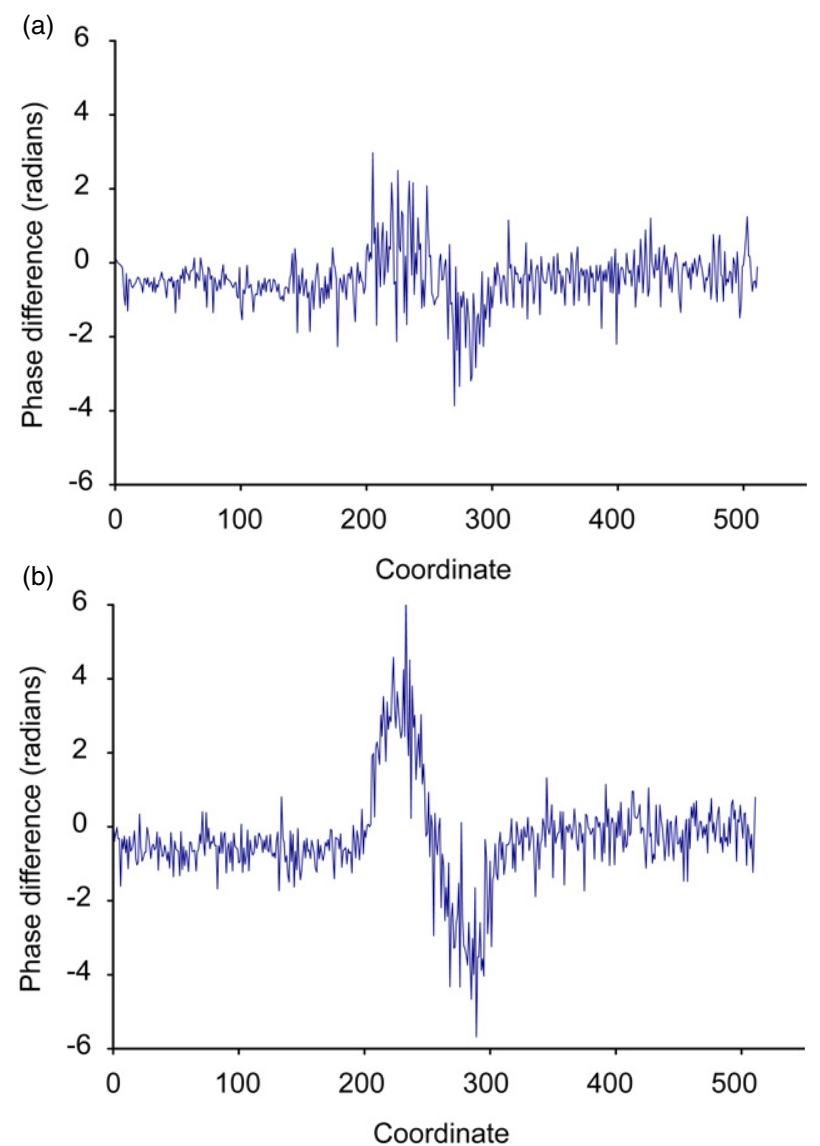

Figure 7. Vertical cross sections for figure 6, (a) before and (b) after modifying the interferometer. Ratio between maximum phase difference and standard deviation of noise is 3.43 before, and 9.91 after, the modification.

shearing, and the intermediate phase differences for a series of measurement intervals were accumulated to obtain an unwrapped final phase difference map. Figure 6(a) shows rather poor results before modifying the interferometer, due to a lack of modulation caused by unbalanced reference and object beams. Figure 6(b) shows much higher quality after the modification. An estimate of the improvement was obtained by calculating the ratio between measured maximum phase difference over a vertical cross section at the defect area and the standard deviation of the noise for that cross section. Before modification this ratio was 3.43; after adding the quarter-wave plate it was 9.91. Figures 7(a) and (b) show the vertical cross sections.

\section{Conclusions}

A polarization-based shearing speckle interferometer has been modified in order to convert predominant unfavourable linear polarization states encountered when a metallic scattering surface is illuminated by a vertically or horizontally polarized beam. As a result linearly polarized light in any direction is converted to elliptically polarized light, with the main axis of the ellipse oriented at $45^{\circ}$, providing well balanced object and reference beams. Average modulation has thus been increased, yielding better $\mathrm{S} / \mathrm{N}$ ratios for measurement 
results. Experimental validation of the proposed method on an unprepared metallic surface has shown that a substantial improvement of results can be obtained for this type of application.

\section{Acknowledgments}

This research was supported by the Technology Foundation STW, Applied Science Division of NWO and the technology program of the Ministry of Economic Affairs. We thank Hans Walhout, Polytechnic of Rijswijk for his contribution to this work.

\section{References}

[1] Francon M and Mallick S 1971 Polarization Interferometers (London: Wiley-Interscience)

[2] Shang H M, Hung Y Y, Luo W D and Chen F 2000 Surface profiling using shearography Opt. Eng. 139 23-31

[3] Hung M Y Y, Long K W and Wang J Q 1997 Measurement of residual stress by phase shift shearography Opt. Lasers Eng. 17 61-73

[4] Debrus S 1977 Speckle shearing interferometer using a Savart plate Opt. Commun. 20 257-61
[5] Nakadate S, Yatagai T and Saito H 1980 Digital speckle-pattern shearing interferometry Appl. Opt. 19 4241-6

[6] Weijers A L, van Brug H and Frankena H J 1998 Polarization phase stepping with a Savart element Appl. Opt. 37 5150-5

[7] Somers P A A M and van Brug H 2001 A single camera, dual image real-time-phase-stepped shearing speckle interferometer Proc. Fringe 2001 (Bremen, 2001) ed W Osten and W Jüptner (Amsterdam: Elsevier) pp 573-80

[8] Goodman J W 1975 Statistical properties of laser speckle patterns Laser Speckle and Related Phenomena (Topics in Applied Physics vol 9) ed J C Dainty (Berlin: Springer)

[9] Adams M, Hinsch K D, Lange F and Wolff K 1997 Polarization effects in speckle correlation metrology Opt. Eng. 36 2225-8

[10] Longhurst R S 1967 Geometrical and Physical Optics (London: Longman Group)

[11] Jones R C 1941 A new calculus for the treatment of optical systems J. Opt. Soc. Am. 31488

[12] Pedrotti F L and Pedrotti L S 1993 Introduction to Optics 2nd edn (Englewood Cliffs, NJ: Prentice-Hall)

[13] Somers P A A M and Bhattacharya N 2005 Maintaining sub-pixel alignment for a single camera two-bucket shearing speckle interferometer J. Opt. A: Pure Appl. Opt. 7 S385-91

[14] Somers P A A M and Bhattacharya N 2005 Polarization plane rotator used as a phase stepping device in a 2-channel shearing speckle interferometer Proc. Optical Metrology (Munich, June 2005); Proc. SPIE 5856 664-73 